\title{
Effect of Ansamitocins on Growth of Yeasts
}

\author{
Seiichi TANIDA and Toru HaSEgawa \\ Microbiological Research Laboratories, Central Research Division, \\ Takeda Chemical Industries, Ltd., 17-85, Jusohonmachi \\ 2-chome, Yodogawa-ku, Osaka 532, Japan
}

Received October 1, 1980

\begin{abstract}
Ansamitocins in combination with amphotericin B produced synergistic inhibition on the growth of several yeasts in liquid cultures. Ansamitocin $\mathrm{P}-3$ at $5 \mu \mathrm{g} / \mathrm{ml}$ completely suppressed the growth of Saccharomyces cerevisiae whereas ansamitocin P-3 alone at $50 \mu \mathrm{g} / \mathrm{ml}$ hardly affected growth. Ansamitocin P-4 and maytansine also showed synergistic activity with amphotericin B against $S$. cerevisiae. The synergism also occurred in cultures of Candida albicans and Hansenula anomala. Combinations of ansamitocin P-3 with various agents revealed that the synergism depended on the specific property of amphotericin $B$. Ansamitocins showed no interfering activity against regeneration of protoplasts of $S$. cerevisias. These results suggest that the limited activity of ansamitocins against these yeasts is due to the membrane permeability barrier of these cells.
\end{abstract}

Ansamitocins, maytansinoid antitumor antibiotics purified from fermentation fluids of Nocardia sp. No. C-15003 (N-1), ${ }^{1,2)}$ have specific actions against microtubule systems in eukaryotic cells, ${ }^{3 \sim 5)}$ resulting in inhibition of growth of several fungi, protozoa and tumor cells, and suppression of the regeneration of cilia in deciliated Tetrahymena at low concentrations. ${ }^{37}$ However, the antibiotics show slight activity against some fungi. ${ }^{2}$ There are two possible reasons for the limited activity of ansamitocins against these fungi, i.e., the low affinity of the antibiotics to the cellular targets in these cells and obstruction by the permeability barrier of their cytoplasmic membrane.

In an attempt to clarify the action of ansamitocins on fungi, we studied the effect of the antibiotics in combination with a polyene antibiotic, amphotericin $B$, against several yeasts tolerant to ansamitocins, and further studies were made using protoplasts of Saccharomyces cerevisiae.

\section{MATERIALS AND METHODS}

Organisms. Saccharomyces cerevisiae IFO 0209, Candida albicans IFO 0583 and Hansenula anomala IFO 0118 were used throughout this work. These yeasts were maintained on agar slants of trypticase soy agar (BBL) containing $1 \%$ glucose.
Synergism studies. The susceptibility of each yeast to ansamitocin $\mathbf{P}-3, P \ldots 4$ or maytansine combined with amphotericin $\mathrm{B}$ or orther agents was determined by the broth dilution method with YMM medium $(0.67 \%$ yeast-nitrogen base (Difco) and $2 \%$ glucose, $\mathrm{pH} 6.5$ ). Each fungus was cultured for 20 hours at $28^{\circ} \mathrm{C}$ with shaking before inoculation. The culture was diluted 10 -fold with the medium and a $50 \mu 1$ portion was transferred to $5 \mathrm{ml}$ of medium with each drug. The initial cell density in this medium was about $5 \times 10^{4} / \mathrm{ml}$. After incubation for 20 hours at $28^{\circ} \mathrm{C}$ with shaking, growth was measured by the turbidity of the culture at $600 \mathrm{~nm}$ with a Hitachi spectrophotometer model 101.

Regeneration of protoplasts. Protoplasts of $S$. cerevisiae were prepared by the method of Yamamoto and Fukui. ${ }^{\text {) }}$ The regeneration of protoplasts was carried out as follows: The protoplastized suspension was diluted with SET medium ${ }^{6)}(1.3 \mathrm{M}$ sorbitol, $0.1 \mathrm{~mm}$ EDTA and $10 \mathrm{~mm}$ Tris-HCl, $\mathrm{pH}$ 7.4), or distilled water as the control, and the diluent was mixed with the regeneration medium kept at $50^{\circ} \mathrm{C}$ which contained $0.5 \%$ yeast extract, $0.5 \%$ peptone, $3 \%$ glucose, $2.5 \%$ agar, and $0.6 \mathrm{M} \mathrm{KCl}, \mathrm{pH} 6.0$. After addition of ansamitocin $\mathrm{P}-3, \mathrm{P}-4$ or maytansine, the mixture was poured into a plastic plate, and then the solidified plate was incubated for 66 hours at $28^{\circ} \mathrm{C}$. The number of regenerated colonies was estimated by subtracting the number of colonies with dilution with distilled water from that with SET.

Chemicals. Ansamitocins P-3 and P-4 were purified from the culture fluid of Nocardia sp. No. C-15003 $(\mathrm{N}-1),{ }^{1,2)}$ Maytansine used in this study was prepared in our research division. Amphotericin B, cyclo- 
heximide, 5-fluorocytosine and deoxycholate were purchased from P-L Biochemicals Inc., USA, Signa Chemials Co., USA, Nippon Roche K.K., Japan, and Difco Co., USA, respectively. Antimycin was purified from the culture fluid of a streptomycete strain isolated in our laboratories.

\section{RESULTS}

Effect of ansamitocins on growth of yeasts in combination with amphotericin $B$

Ansamitocins showed no activity against several yeasts at concentrations up to $50 \mu \mathrm{g} / \mathrm{ml}$. In an attempt to clarify whether their limited antifungal activity is due to the permeability barrier of the membrane of the tolerant cells, combination of ansamitocin $\mathrm{P}-3$ with a polyene antibiotic, amphotericin $\mathrm{B}$, was examined. As shown in Fig. 1, ansamitocin P-3 at $5 \mu \mathrm{g} / \mathrm{ml}$ in combination with amphotericin $B$ at 0.05 $\mu \mathrm{g} / \mathrm{ml}$ showed complete inhibition of growth of S. cerevisiae, whereas ansamitocin $\mathrm{P}-3$ at 20 $\mu \mathrm{g} / \mathrm{ml}$ alone showed no inhibition. Combination of ansamitocin $\mathrm{P}-3$ with amphotericin $\mathrm{B}$ at $0.02 \mu \mathrm{g} / \mathrm{ml}$ or less exhibited no marked effect. When amphotericin B was added to the exponential culture in the presence of ansamitocin

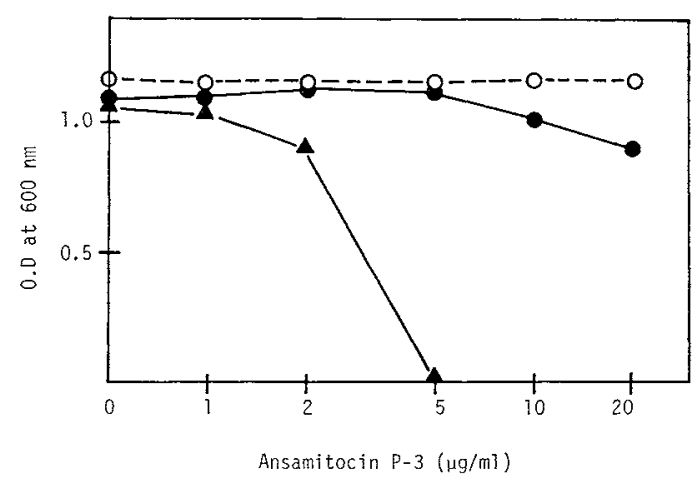

FIG. 1. Inhibition of Growth of $S$. cerevisiae by Ansamitocin $\mathrm{P}-3$ in Combination with Amphotericin B.

A 20 hour culture was diluted 10 -fold with fresh medium and a $50 \mu \mathrm{l}$ portion was transferred to $5 \mathrm{ml}$ of medium with varying concentrations of ansamitocin P-3 and amphotericin B. After incubation for 20 hours at $28^{\circ} \mathrm{C}$, the turbidity of the culture at $600 \mathrm{~nm}$ was measured. $O$, without amphotericin $\mathrm{B} ; \mathbf{0}$, amphotericin $\mathbf{B}$ at $0.02 \mu \mathrm{g} / \mathrm{ml} ; \mathbf{A}$, amphotericin $\mathbf{B}$ at $0.05 \mu \mathrm{g} / \mathrm{ml}$.

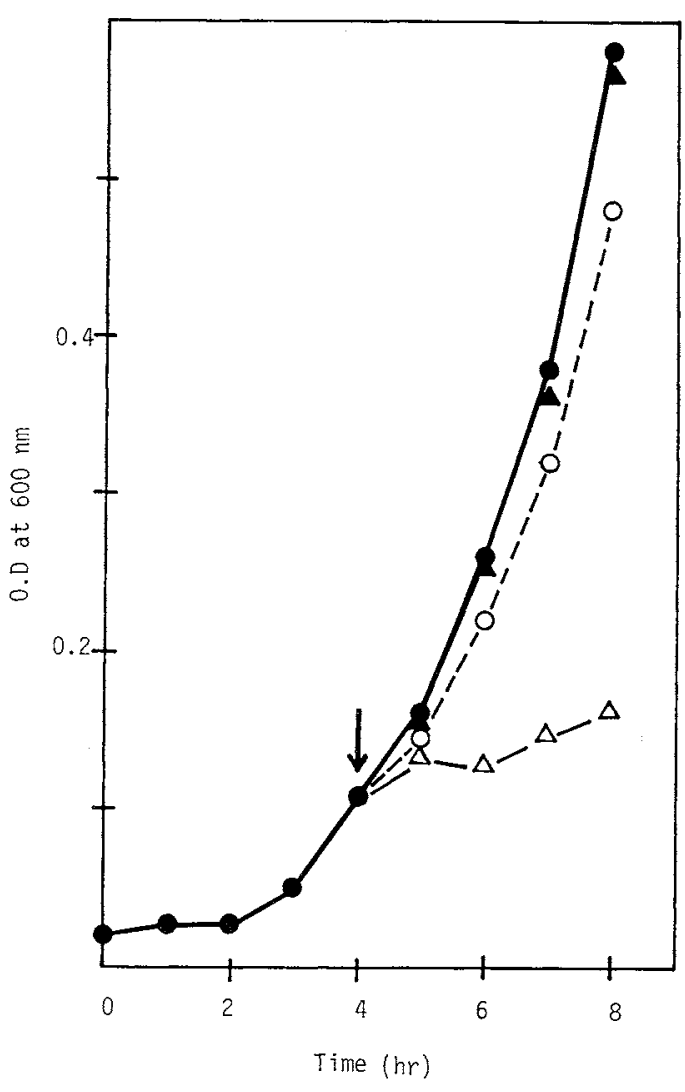

FIG. 2. Inhibition of Exponential Growth of $S$. cerevisiae by Ansamitocin $\mathrm{P}-3$ in Combination with Amphotericin B.

Ansamitocin $\mathrm{P}-3$ at $10 \mu \mathrm{g} / \mathrm{ml}$ was added at time zero. The arrow indicates the addition of amphotericin $\mathbf{B}$ at $0.05 \mu \mathrm{g} / \mathrm{ml}$. - , no drug; $\mathbf{A}$, ansamitocin $\mathrm{P}-3$ alone; $\mathrm{O}$, amphotericin $\mathrm{B}$ alone; $\triangle$, ansamitocin $\mathrm{P}-3$ plus amphotericin B.

P -3 at $10 \mu \mathrm{g} / \mathrm{ml}$, growth was prevented after one hour of the subsequent division (Fig. 2.) Ansamitocin P-4 and maytansine, which has a structure ${ }^{7)}$ and function ${ }^{8 \sim 12)}$ closely related to ansamitocins, also showed synergistic inhibition with amphotericin B against $S$. cerevisiae (Fig. 3). The inhibitory activity of ansamitocin P-3 and maytansine combined with amphotericin $\mathrm{B}$ at $0.05 \mu \mathrm{g} / \mathrm{ml}$ was weaker than that of ansamitocin P-4. Ansamitocins at $20 \mu \mathrm{g} / \mathrm{ml}$ alone did not affect the growth of Hansenula anomala or Candida albicans. However, the growth of both organisms was completely suppressed by ansamitocin $\mathrm{P}-3$ at $5 \mu \mathrm{g} / \mathrm{ml}$ in 


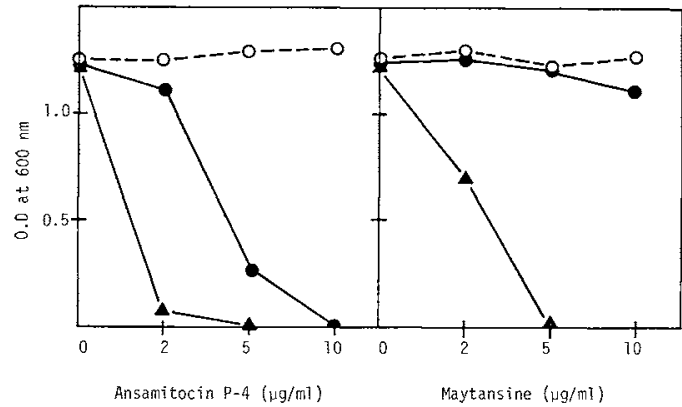

FrG. 3. Inhibition of Growth of $S$. cerevisiae by Ansamitocin P-4 and Maytansine in Combination with Amphotericin B.

Procedures and symbols are the same as described in Fig. 1.

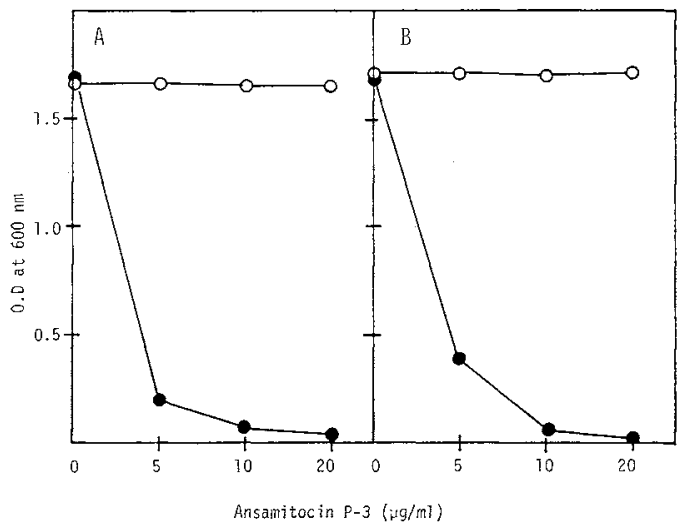

FIG. 4. Inhibition of Growth of C. albicans and H. anomala by Ansamitocin P-3 in Combination with Amphotericin B.

Procedures are the same as described in Fig. 1. A, C. albicans IFO 0583; B, H. anomala IFO 0118. $O$, without amphotericin $\mathrm{B}$; $0.05 \mu \mathrm{g} / \mathrm{ml}$.

combination with amphotericin B at $0.05 \mu \mathrm{g} / \mathrm{ml}$ (Fig. 4).

In order to clarify the specific property of amphotericin B, combinations of ansamitocin P-3 with other agents with different action mechanisms were examined. As shown in Fig. 5, cycloheximide, a specific inhibitor of protein synthesis in eukaryotic cells, and 5fluorocytocine, an inhibitor of nucleic acid synthesis in fungal cells, showed no synergistic effect. Antimycin, an inhibitor of the respiratory mechanism, and deoxycholate, a surface activating agent, caused a synergistic effect

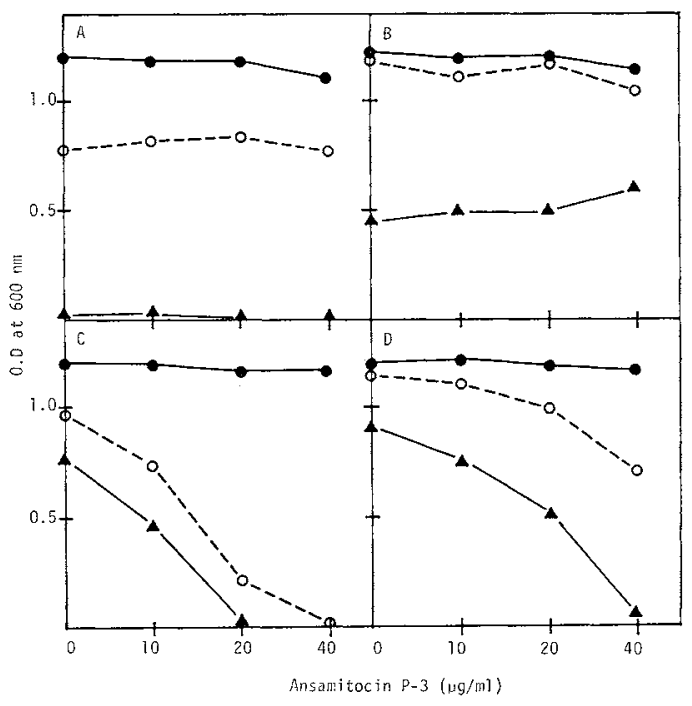

FIG. 5. Inhibition of Growth of $S$. cerevisiae by Ansamitocin P-3 in Combination with Various Agents.

Procedures are the same as described in Fig. 1. A: - without cycloheximide $(\mathrm{CYH}) ; 0, \mathrm{CYH}$ at $0.02 \mu \mathrm{g} / \mathrm{ml} ; \Delta, \mathrm{CYH}$ at $1.0 \mu \mathrm{g} / \mathrm{ml}$. B: $\boldsymbol{0}$, without 5-fluorocytosine $(5 \mathrm{FC}) ; 0,5 \mathrm{FC}$ at $0.005 \mu \mathrm{g} / \mathrm{ml} ; \boldsymbol{\Lambda}$, $5 \mathrm{FC}$ at $0.02 \mu \mathrm{g} / \mathrm{ml} . \quad \mathrm{C}$ : $\bullet$, without antimycin (ANM); O, ANM at $0.5 \mu \mathrm{g} / \mathrm{ml} ; \Delta, A N M$ at $1.0 \mu \mathrm{g} / \mathrm{ml}$. D: without deoxycholate (DOC); O, DOC at $20 \mu \mathrm{g} / \mathrm{ml}$; $\Delta$, DOC at $40 \mu \mathrm{g} / \mathrm{ml}$.

with ansamitocin $\mathrm{P}-3$, but to a much lesser extent than amphotericin $\mathrm{B}$. These results suggest that the synergism depends on the specific property of amphotericin B.

\section{Effect of ansamitocins on the regeneration of protoplasts of S. cerevisiae}

If ansamitocins cannot penetrate the membrane of tolerant fungi, the regeneration of protoplasts into vegetative cells in these organisms might not be affected. In fact, the regeneration of protoplasts of $S$. cerevisiae to form colonies was hardly affected by ansamitocins (Table I). Although the growth was completely suppressed by $5 \mu \mathrm{g} / \mathrm{ml}$ of ansamitocin P-3 combined with $0.05 \mu \mathrm{g} / \mathrm{ml}$ of amphotericin B (Fig. 1), a high regeneration value was obtained even at $100 \mu \mathrm{g} / \mathrm{ml}$ of ansamitocin P-3 alone. Ansamitocin P-4 and maytansine also showed only slight activity against the regeneration of protoplasts at a 
Table I. Effect of Ansamitocins on the Regeneration of Yeast Protoplasts

Protoplasts were prepared from $S$. cerevisiae IFO 0209 by the method of Yamato and Fukui. ${ }^{6)}$ The regeneration of protoplasts was carried out by the method described in MATERIAIS AND METHODS. The number of regenerated colonies was estimated by subtracting the number of colonies with difution with distilled water from that with SET medium. The value of relative regeneration was estimated relative to the number of regenerated colonies without ansamitocins, which was taken as 1.0.

\begin{tabular}{lcccc} 
Compound & Concn. & $\begin{array}{c}\text { Total } \\
\text { colonies }\end{array}$ & $\begin{array}{c}\text { Regenerated } \\
\text { colonies }\end{array}$ & $\begin{array}{c}\text { Relative } \\
\text { regeneration }\end{array}$ \\
\hline Ansamitocin P-3 & $0 \mu \mathrm{g} / \mathrm{ml}$ & $1.18 \times 10^{8} / \mathrm{ml}$ & $9.19 \times 10^{7} / \mathrm{ml}$ & 1.00 \\
& 1 & 1.13 & 8.72 & 0.95 \\
& 10 & 1.06 & 8.36 & 0.91 \\
Ansamitocin P-4 & 100 & 0.72 & 4.66 & 0.51 \\
Maytansine & 10 & 1.12 & 8.12 & 0.88 \\
\hline
\end{tabular}

concentration of $10 \mu \mathrm{g} / \mathrm{ml}$, the same as ansamitocin P-3. It is therefore clear that ansamitocins and maytansine can hardly penetrate the membrane of $S$. cerevisiae and have no interfering activity against the formation of the cell wall in this organism.

\section{DISCUSSION}

Ansamitocins are specific inhibitors for microtubule systems in eukaryotic cells. Although some eukaryotic microorganisms show tolerance to ansamitocins, ${ }^{2}$ ) their mitotic process might also be susceptible to the antibiotics because of the closely related properties of tubulin molecules among higher and lower eukaryotic organisms. ${ }^{13)}$ As shown in this communication, ansamitocins show a synergistic effect with amphotericin B against some yeasts that are relatively resistant to ansamitocins when used alone. The polyene antibiotics, such as amphotericin B, are well-known to interact with sterol-containing membranes of eukaryotic cells ${ }^{14}$ resulting in leakage of cellular constituents and permeability changes. ${ }^{15 \sim 18)}$ Therefore, the basis of this synergism is most likely amphotericin B-induced penetration of ansamitocins into these tolerant yeasts, resulting in the inhibition of the functions of their microtubule systems. In the same way the antifungal property of maytansine may be enhanced by amphotericin B. This presumption is supported by the results which show that ansamitocins and maytansine did not suppress the regeneration of protoplasts of
S. cerevisiae into vegetative cells. This observation indicates that these compounds can neither penetrate the membrane of the yeast nor interfere with the formation process of its cell wall.

Agents for the specific disruption of the mitotic apparatus are available for studying the relationship between growth and nuclear division in fungi. For instance, colcemid was used for investigating the growth of yeasts. ${ }^{19 \sim 21)}$ Since ansamitocins have specific characters that inhibit mitosis resulting in metaphase arrest of susceptible cells, ${ }^{1,4)}$ combinations of ansamitocins with amphotericin B may also be useful for studying nuclear division in fungi.

\section{REFERENCES}

1) E. Higashide, M. Asai, K. Ootsu, S. Tanida, Y. Kozai, T. Hasegawa, T. Kishi, Y. Sugino and M. Yoneda, Nature, 270, 721 (1977).

2) S. Tanida, T. Hasegawa, K. Hatano, E. Higashide and M. Yoneda, J. Antibiotics, 33, 192 (1980).

3) S. Tanida, E. Higashide and M. Yoneda, Antimicrobial Agents and Chemother., 16, 101 (1979).

4) K. Ootsu, Y. Kozai, M. Takeuchi, S. Ikeyama, K. Tgarashi, K. Tsukamoto, Y. Sugino, T. Tashiro, S. Tsukagoshi and Y. Sakurai, Cancer Res., 40, 1707 (1980).

5) S. Tanida, E. Higashide and M. Yoneda, J. Gen. Microbiol., 118, 411 (1980).

6) M. Yamamoto and S. Fukui, Agric. Biol. Chem., 41, 1829 (1977).

7) S. M. Kupchan, Y. Komoda, W. A. Court, G. J. Thomas, R. M. Smith, A. Karim, C. J. Gilmore, R. C. Haltiwanger and R. F. Bryan, J. Am. Chem. Soc., 94, 1354 (1972). 
8) S. Remillard, L. I. Rebhun, G. A. Howie and S. M. Kupchan, Science, 189, 1002 (1975).

9) M. K. Wolpert-DeFilippes, V. H. Bono, Jr., R. L. Dion and D. G. Johns, Biochem. Pharmacol., 24, 1735 (1975).

10) F. Mandelbaum-Shavit, M. K. Wolpert-DeFilippes and D. G. Johns, Biochem. Biophys. Res. Commun., 72, 47 (1976).

11) B. Bhattacharyya and J. Wolf, FEBS Lett., 75, 159 (1977).

12) R. K. Johnson, T. Inouye and M. K. WolpertDeFilippes, Biochem. Pharmacol., 27, 1973 (1978).

13) R. E. Stephens and K. T. Edds, Physiological Reviews, 56, 709 (1976).

14) J. M. T. Hamilton-Miller, Adv. Appl. Microbiol., 17, 109 (1974).
15) G. Medoff, G. S. Kobayashi, C. N. Kwan, D. Schlessinger and P. Venkov, Proc. Natl. Acad. Sci. U. S., 69, 196 (1972).

16) G. S. Kobayashi, S. C. Cheung, D. Schlessinger and G. Medoff, Antimicrobial Agents and Chemother., 5, 16 (1974).

17) W. C. Chen, D. Chou and D. S. Feingold, Antimicrobial Agents and Chemother., 13, 914 (1978).

18) J. Palacios and R. Serrano, FEBS Lett., 91, 198 (1978).

19) S. Lederberg and G. Stetten, Science, 168, 485 (1970).

20) J. E. Haber, J. G. Peloquin, H. O. Halvorson and G. G. Borisy, J. Cell Biol., 55, 355 (1972).

21) S. Lederberg, R. L. Gourse and D. L. Sackett, $J$. Bacteriol., 129, 198 (1977). 